\title{
Characterization of glomerular diseases using proteomic analysis of laser capture microdissected glomeruli
}

\author{
Anjali A Satoskar ${ }^{1, *}$, John P Shapiro ${ }^{2, *}$, Cherri N Bott ${ }^{1}$, Huijuan Song ${ }^{3}$, \\ Gyongyi M Nadasdy ${ }^{1}$, Sergey V Brodsky ${ }^{1}$, Lee A Hebert ${ }^{3}$, Daniel J Birmingham ${ }^{3}$, \\ Tibor Nadasdy ${ }^{1}$, Michael A Freitas ${ }^{2, \dagger}$ and Brad H Rovin ${ }^{3, \dagger}$ \\ ${ }^{1}$ Department of Pathology, The Ohio State University Medical Center, Columbus, OH, USA; ${ }^{2}$ Department \\ of Molecular Virology, Immunology and Medical Genetics, The Ohio State University Medical Center, \\ Columbus, OH, USA and ${ }^{3}$ Department of Internal Medicine, Division of Nephrology, The Ohio State \\ University Medical Center, Columbus, OH, USA
}

\begin{abstract}
The application of molecular techniques to characterize clinical kidney biopsies has the potential to provide insights into glomerular diseases that cannot be revealed by traditional renal pathology. The present work is a proof-of-concept approach to test whether proteomic analysis of glomeruli isolated from clinical biopsies by laser capture microdissection can provide unique information regarding differentially expressed proteins relevant to disease pathogenesis. The proteomes of glomeruli isolated by laser capture microdissection from biopsies of normal kidneys (living-related donor kidneys) were compared with those from patients with diabetic nephropathy, lupus nephritis, and fibronectin glomerulopathy. Glomerular proteins were extracted, trypsin digested, and subjected to liquid chromatography-tandem mass spectrometry for identification and quantitation. Relative to normal glomeruli, all disease-associated glomeruli showed an increased presence of complement components, a marked decline in podocyte-associated proteins, and a decrease in proteins associated with cellular metabolism. Additionally, fibronectin glomerulopathy glomeruli differed from all the other glomeruli because of a significant accumulation of fibronectin and fibulin. This study demonstrates that our method acquires reproducible and quantitative proteomic information from laser capture microdissection isolates that can be used to characterize the molecular features of glomerular diseases. Modern Pathology (2012) 25, 709-721; doi:10.1038/modpathol.2011.205; published online 27 January 2012
\end{abstract}

Keywords: glomerular disease; kidney; laser capture microdissection; lupus; mass spectrometry; proteomics

The application of molecular techniques such as genomics, proteomics, or metabolomics to clinical kidney biopsies has the potential of providing significantly more new and unique information regarding glomerular diseases than traditional renal pathology using microscopy. New insights could be relevant to glomerular disease pathogenesis, novel therapeutic targets, or inter-patient variability. While there have

Correspondence: Dr BH Rovin, Division of Nephrology, Ohio State University, 395 W. 12th Avenue, Ground Floor, Columbus, OH, USA.

E-mail: Rovin.1@osu.edu

*These authors contributed equally to this work.

†These authors contributed equally to this work as senior authors.

Received 12 August 2011; revised 17 October 2011; accepted 17 October 2011; published online 27 January 2012 been several reports on the use of laser capture microdissection in the analysis of glomerular diseases, these investigations mainly focused on changes in gene expression in specific kidney compartments. ${ }^{1-6}$ A small number of studies have tested the feasibility of proteomic analysis of isolated glomeruli in animal models ${ }^{7,8}$ or human tissue. ${ }^{9-11}$ Only one investigation showed that laser capture microdissection glomeruli from a clinical kidney biopsy specimen yielded sufficient material to create a robust proteome. However, none of the proteins was further characterized. ${ }^{10}$

The present work was undertaken to more clearly establish whether proteomic analysis of laser capture microdissection glomeruli from clinical kidney biopsies can be used to identify differentially expressed glomerular proteins that may inform disease 
pathogenesis. For this proof-of-concept study, we feature an examination of laser capture microdissection glomeruli from patients with diabetic nephropathy, lupus nephritis, and fibronectin glomerulopathy. Fibronectin glomerulopathy is a rare disease with large, proteinaceous glomerular deposits, which to date have only been assessed by traditional immunohistochemistry. Thus, it is an ideal clinical model to evaluate the extent to which analysis by proteomics can exceed that of immunohistologic methods.

Fibronectin glomerulopathy is a familial glomerulopathy characterized clinically by proteinuria, microscopic hematuria, hypertension, and progressive renal dysfunction, which can lead to end-stage renal disease. ${ }^{12}$ An autosomal dominant inheritance pattern with age-related penetrance affecting both genders has been demonstrated. ${ }^{13,14}$ Histologically, fibronectin glomerulopathy is characterized by lobular glomerular enlargement, obliteration of capillary loops, and mesangial expansion due to accumulation of acellular material. These deposits are large homogenous to finely granular electron dense masses, usually with no distinctive fibrillary substructure. By immunohistochemical staining, the glomerular material stains for fibronectin ${ }^{15}$ and only weakly or not at all for IgG, complement products, or other matrix components like laminin and collagen type IV. ${ }^{15}$ Using antibodies to plasma-derived and cell-derived fibronectin, it has been reported that the fibronectin in the deposits is mainly plasma derived. ${ }^{15,16}$ Therefore, it has been postulated that fibronectin glomerulopathy is a primary mesangiopathic glomerulopathy that is caused by abnormal plasma fibronectin deposition, and subsequent disruption of the glomerular architecture and filtration barrier leading to glomerular proteinuria and loss of glomerular filtration rate.

However, fibronectin deposition is not specific for fibronectin glomerulopathy. Intra-glomerular fibronectin can be found in other kidney diseases, such as diabetic nephropathy and lupus nephritis. ${ }^{17-19}$ These findings suggested that comparing glomerular protein, expression of fibronectin glomerulopathy to that of normal kidneys, and kidneys with diabetic nephropathy and lupus nephritis would be a good test for demonstrating the capability of using laser capture microdissection in combination with liquid chromatography-tandem mass spectrometry to characterize kidney diseases in greater detail. The approach used here revealed anticipated differences as well as unexpected similarities between disease states, and also highlighted potential pathogenic mechanisms not previously known or considered for these diseases.

\section{Materials and methods}

\section{Kidney Tissue}

Baseline allograft biopsies from living donor renal allograft recipients $(n=3)$ were used as normal controls. Glomerular disease biopsies included diabetic nephropathy $(n=2)$, lupus nephritis proliferative class IV $(n=3)$, and pure membranous class V $(n=2)$, and two patients from a family known to have fibronectin glomerulopathy. The kidney biopsy findings of this family have been previously reported. ${ }^{20}$ One biopsy was from an affected native kidney, while the second biopsy was from a livingunrelated donor renal allograft that developed recurrent disease 3 years post-transplant. The study was approved by the Ohio State University Institutional Review Board (Protocol number 2009H0179).

\section{Laser Capture Microdissection and Mass Spectrometry}

Laser capture microdissection was performed using the laser microdissection system from PALM Technologies (Carl Zeiss MicroImaging $\mathrm{GmbH}$, Munchen, Germany) containing a PALM MicroBeam and RoboStage for high-throughput sample collection and PALM RoboMover (PALM Robo software, Version 2.2). Typical settings used for laser cutting were UVEnergy of 52 and UV-Focus of 79-81. Laser capture microdissection was done using snap frozen tissue embedded in optimum cutting temperature medium at the time of the biopsy. The frozen tissue blocks were cut into $10 \mu \mathrm{m}$ sections, mounted on a thermoplastic (polyethylenenapthalate) covered glass slide (Carl Zeiss MicroImaging) and kept at $-80^{\circ} \mathrm{C}$ until use. When needed, sections were briefly thawed and immediately fixed in ice cold $70 \%$ ethanol and rinsed in deionized water for $1 \mathrm{~min}$. After the water rinse, the wet sections were stained briefly (5-10 s) with hematoxylin, immediately rinsed, and then dehydrated in graded ethanol. The dehydrated slides were allowed to air dry for immediate capture on the PALM technology microdissection system.

Sections were cut and captured under a $\times 10$ ocular lens. Fifteen $10 \mu \mathrm{m}$ sections from each clinical biopsy case were utilized, and each section contained an average of 10 glomeruli. The glomeruli were isolated by dissecting around Bowman's capsule. For each biopsy, an average of 75 glomerular cross-sections, equivalent to about 7000 cells were captured. Cut elements were catapulted into $25 \mu \mathrm{l}$ of $0.5 \%$ Rapigest (Waters Corporation, MA, USA), resuspended in $50 \mathrm{mM} \mathrm{NH} \mathrm{NCO}_{3}$, collected in a $0.2-\mathrm{ml}$ tube, and held at $-80^{\circ} \mathrm{C}$ until protein retrieval.

For protein retrieval, samples that had been stored at $-80^{\circ} \mathrm{C}$ were thawed briefly and sonicated (in the original $0.2 \mathrm{ml}$ tube) on the low power setting for a total instrument time of $60 \mathrm{~s}$ with a $3 \mathrm{~s}$ on $3 \mathrm{~s}$ off time interval using a Diagenode Bioruptor (model UCD200, NJ, USA). The samples were placed in a boiling water bath for $5 \mathrm{~min}$ and cooled to room temperature. Trypsin was added in a ratio of 1:30 trypsin:protein assuming $\sim 2 \mu \mathrm{g}$ retrieved protein/10000 isolated cells. After overnight incubation at $37^{\circ} \mathrm{C}$ with gentle shaking, formic acid was added to a final concentration of $30 \%$ and the suspension was 
incubated for $30 \mathrm{~min}$ at $37^{\circ} \mathrm{C}$ to precipitate the Rapigest. The extracts were dried and the protein digests were resuspended in $20 \mu \mathrm{l}$ of a solution of $2 \%$ acetonitrile with $0.1 \%$ formic acid and sonicated for 1-2 min in a water bath sonicator at $4^{\circ} \mathrm{C}$ to ensure peptide solubilization. Peptide/protein concentration was obtained at $280 \mathrm{~nm}$ absorbance using $1 \mu \mathrm{l}$ of sample on a Nanodrop ND-1000 spectrometer.

For mass spectrometry analysis, peptides were separated by reversed-phase HPLC (Dionex Ultimate 3000 capillary/nano HPLC system, Dionex, CA, USA) and mass analyzed with a Thermo Fisher LTQ Orbitrap XL (CA, USA), equipped with micro/ nanospray ionization sources (Michrom Bioresources, Auburn, CA, USA). HPLC separations were carried out at a flow rate of $2 \mu \mathrm{l} / \mathrm{min}$ on a $0.2 \mathrm{~mm} \times$ $150 \mathrm{~mm}$ C18 column ( $5 \mu \mathrm{m}, 300 \AA$; Michrom Bioresources). The mobile phases consisted of HPLC grade water and acetonitrile with formic acid added as ion-pairing reagent. A $10-32 \%$ gradient was run over $5 \mathrm{~h}$ followed by a $32-90 \%$ gradient run over $40 \mathrm{~min}$. The heated capillary temperature and electrospray voltage were set at $175^{\circ} \mathrm{C}$ and $2.0 \mathrm{kV}$, respectively. Protein identifications were obtained using the MassMatrix search engine. ${ }^{21-24}$ An average of 350 proteins were identified per biopsy, with a range of 250-450 proteins.

\section{Immunoperoxidase Staining}

Immunoperoxidase staining with a monoclonal antibody to fibulin-1 (Santa Cruz Biotechnology, CA, USA) and a polyclonal antibody to podocin (Alpha Diagnostic Intl, TX, USA) was performed (dilution $1: 100$ and 1:40, respectively) on formalin-fixed paraffin-embedded tissue sections of the fibronectin glomerulopathy biopsies, and additional cases of diabetic nephropathy and proliferative class IV lupus nephritis (different from the cases used for mass spectrometry). Fibronectin immunostaining was performed in the laboratory of the late Dr Andrew Herzenberg, Toronto, Canada). The antibody used was a mouse monoclonal antibody directed to the $\mathrm{N}$-terminal of fibronectin. Control tissues used to validate the antibodies were normal kidney, aorta, placenta, and blood vessel walls in the gastrointestinal tract.

\section{DNA Sequencing}

Genomic DNA samples were sequenced for fibronectin gene coding region exons 19,36 , and 37 , and the complete coding region, including intron-exon boundaries for the fibulin-1 gene. Genomic DNA was extracted from peripheral blood leukocytes using Gentra Puregene Blood Kits (Qiagen, CA, USA) or frozen serum using the QIAamp Investigator kit (Qiagen). DNA amplification was carried out by polymerase chain reaction (PCR). Oligonucleotide primers (Invitrogen, CA, USA) were specifically designed to flank fibronectin exons 19, 36, and 37, and fibulin-1 exons 1-20, according to the gene bank published sequences. Primer sequences are available upon request. PCR products ranged in size from 262 to 983 basepairs.

PCR products were checked on $2 \%$ agarose gel to confirm a single product for direct sequencing with BigDye Terminator Cycle Sequencing Chemistry (Applied Biosystems, CA, USA) using the same PCR primers. Sequencing was performed by 3730 DNA Analyzer by Applied Biosystems.

\section{Data and Statistical Analysis}

Protein quantitation was performed by analysis of peptide spectral counts for each protein group identified. ${ }^{25-27}$ The MassMatrix protein identification search results were regrouped by use of an in-house developed application to create a parsimonious protein list. ${ }^{28}$ This application regroups protein identifications based on common peptides observed in all sample data sets. Hierarchical clustering of the total peptide matches for each protein group was then performed using Cluster 3.0. The Ingenuity Pathway Analysis tool was used to examine glomerular molecular pathways based on identified proteins (Ingenuity Systems; http://www.ingenuity. com). To evaluate statistically significant difference in protein abundances, the MS/MS data for each sample classification were merged, analyzed by MassMatrix and combined into a parsimonious protein list. The resulting peptide spectral counts for each protein were then normalized by linear scaling such that the total spectral counts were equal. Sample classes were then evaluated pairwise using the $G$-test to determine if differences in spectral counts were significant. ${ }^{29}$

\section{Results}

The pedigree of the fibronectin glomerulopathy family is shown in Figure 1a, and the two family members whose biopsies were studied by laser capture microdissection/proteomics are indicated. It has previously been shown that several fibronectin glomerulopathy kindreds display an $A \rightarrow G$ singlenucleotide polymorphism in heparin-binding domain III of exon 19 of the fibronectin gene. ${ }^{30}$ This results in a tyrosine $\rightarrow$ cysteine amino-acid change, and may provide a genetic predisposition for fibronectin glomerulopathy. Exon 19 of the fibronectin gene from our patients was sequenced and showed this same heterozygous $A \rightarrow G$ singlenucleotide polymorphism (Figure 1b). Another affected and one unaffected family member were also sequenced, and the results are shown in Figure 1a.

Renal biopsies from both fibronectin glomerulopathy patients showed prominent glomerular enlargement and diffuse acellular mesangial expansion that was periodic acid-schiff positive and 
a

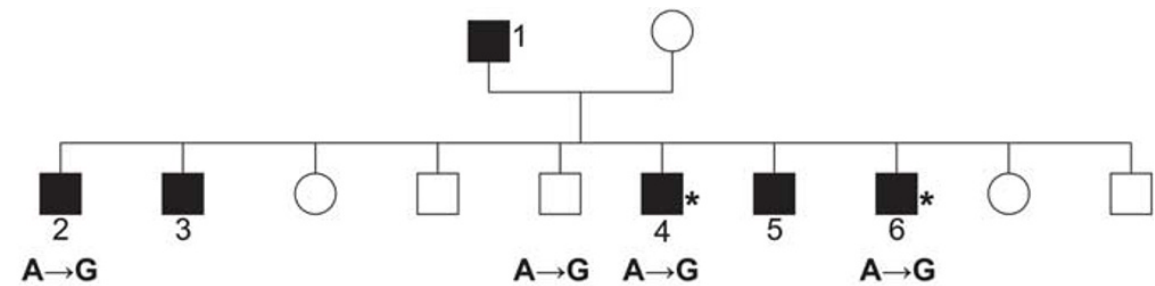

1. At age 64 proteinuria, hematuria discovered on routine physical, declined biopsy

2. Received kidney transplant from unaffected sibling at age 18 ; proteinuria $1.6 \mathrm{~g} / \mathrm{d}$ and serum creatinine $177 \mu \mathrm{mol} / \mathrm{L}$

3. First kidney transplant at age 37 , second transplant age 53; proteinuria $0.7 \mathrm{~g} / \mathrm{d}$ and serum creatinine $230 \mu \mathrm{mol} / \mathrm{L}$

4. Proteinuria $1.3 \mathrm{~g} / \mathrm{d}$ and serum creatinine $106 \mu \mathrm{mol} / \mathrm{L}$

5. Serum creatinine $212 \mu \mathrm{mol} / \mathrm{L}$

6. Received kidney transplant from wife at age 43 , with recurrent FNG after 3 years; proteinuria $2.8 \mathrm{~g} / \mathrm{d}$ and serum creatinine $265 \mu \mathrm{mol} / \mathrm{L}$

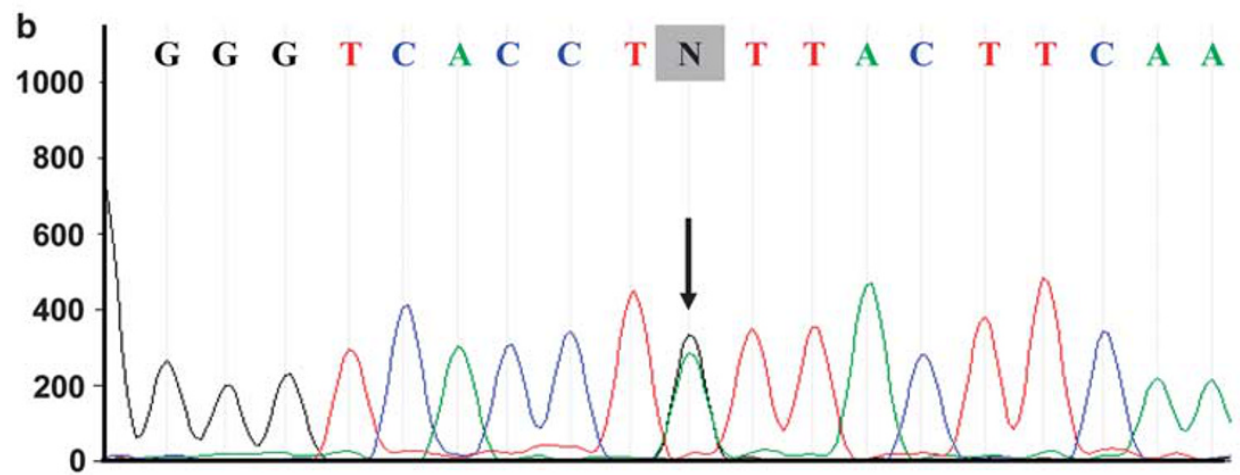

Figure 1 Pedigree and genetic analysis of a family with fibronectin glomerulopathy. (a) Affected members are indicated by filled symbols. Males are squares, females are circles. The patients whose biopsies were analyzed by laser capture microdissection/proteomics are indicated with an '*'. The results of genotyping the fibronectin gene FN1 are indicated below those family members who provided DNA for analysis. (b) Example of DNA sequencing of one of the patients showing the common heterozygous A $\rightarrow$ G single-nucleotide polymorphism.

methenamine silver negative (Figure 2a and b). On ultrastructural examination, the glomeruli showed bulky, nondescript granular electron dense material in the mesangium and in the widened subendothelial space along glomerular capillary walls (Figure 2c). Direct immunofluorescence staining for IgG, IgM, IgA, and C3 did not reveal strong specific staining for any of these immunoreactants. The bulky glomerular deposits did, however, show staining with the antibody to fibronectin (Figure 2d).

A heat map of glomerular protein expression obtained from the liquid chromatography-tandem mass spectrometry analysis of the glomerular laser capture microdissection isolates is shown in Figure 3. The two patients with fibronectin glomerulopathy were most closely clustered, followed by the three normal controls. There was more variability among the patients with lupus nephritis and diabetic nephropathy. Compared with normal glomeruli, fibronectin glomerulopathy glomeruli displayed 133 differentially expressed proteins that achieved a predetermined level of significance ( $G$-score $\geq 5)$. A subset of these differentially expressed proteins is shown in Table 1. The normalized spectral ratios show the fold-increase (or decrease) of these proteins relative to normal. Table 2 shows the raw spectral counts for each of these proteins and for each individual normal and fibronectin glomerulopathy patient. For comparison, the same proteins were also examined for differential expression relative to normal glomeruli in diabetic nephropathy and lupus nephritis (Table 1). The most striking difference between fibronectin glomerulopathy and normal glomeruli was the extensive level of fibronectin expression in the affected glomeruli. Diabetic nephropathy and lupus nephritis did not show any increase in glomerular fibronectin over control. Additionally, fibulin-1 and fibulin-5 were highly abundant in fibronectin glomerulopathy, and were not found or were minimally detected in normal glomeruli, or glomeruli 

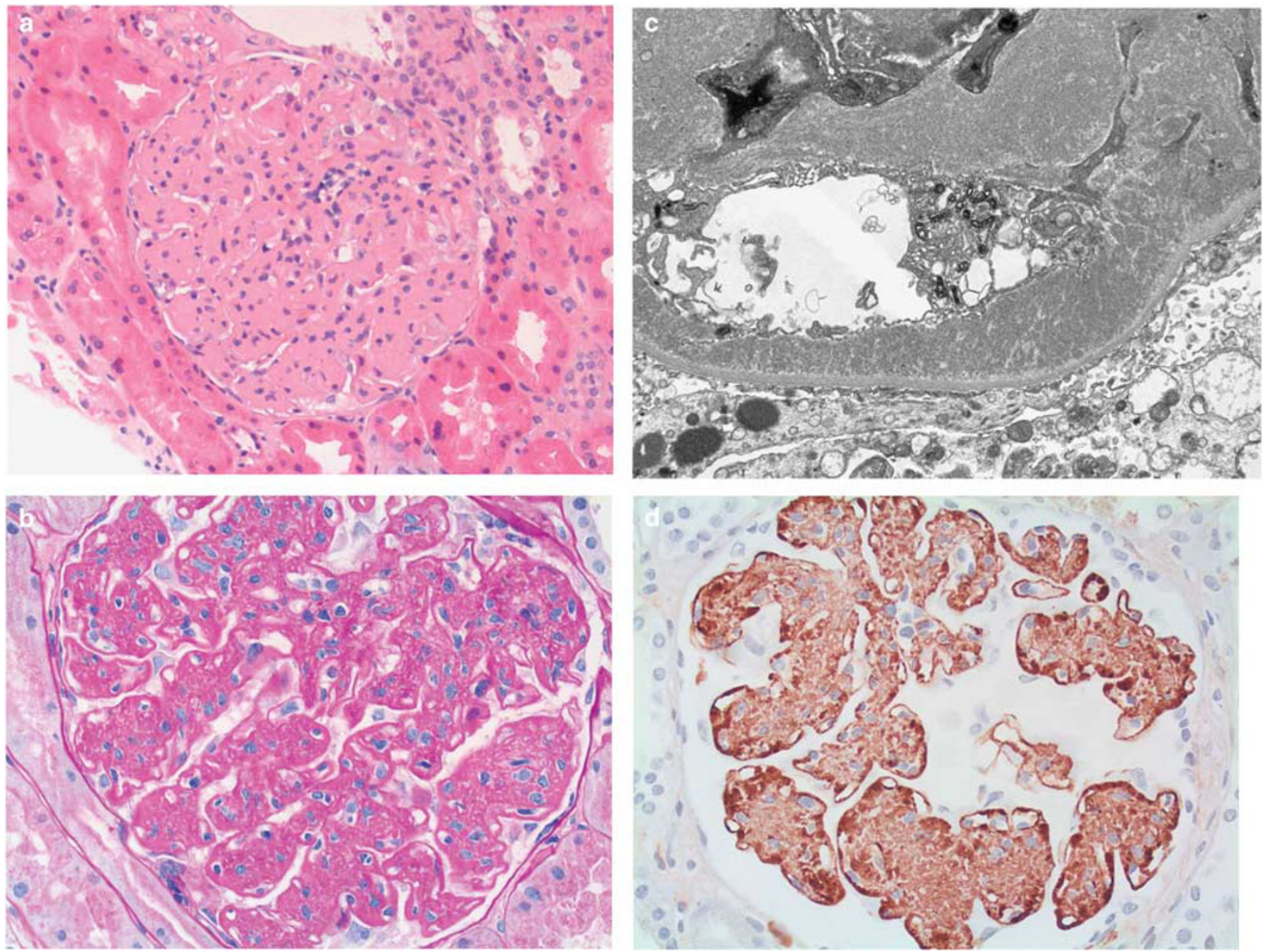

Figure 2 Recurrent fibronectin glomerulopathy in renal allograft of Patient 6. (a) Hematoxylin and eosin-stained tissue showing enlarged glomeruli and acellular expansion of mesangium $(\times 200)$. (b) Mesangial deposits stain with periodic acid-schiff $(\times 400)$. $(\mathbf{c})$ Bulky deposits as seen on electron microscopic examination (uranyl acetate and lead citrate staining). (d) Immunoperoxidase staining for fibronectin with mouse monoclonal antibody to N-terminal of fibronectin shows strong global mesangial positivity $(\times 400)$.

from patients with diabetic nephropathy and lupus nephritis.

A number of other proteins were altered in fibronectin glomerulopathy, diabetic nephropathy, and lupus nephritis. Unexpectedly, many components of the complement pathway were found in fibronectin glomerulopathy and diabetic nephropathy, diseases not associated with systemic complement activation or consumption. Glomeruli from biopsies with both class IV and class V lupus nephritis showed complement expression, as expected. In all the disease groups, podocyte-associated proteins and anti-oxidant proteins were markedly decreased relative to control. Several serum proteins were increased in fibronectin glomerulopathy, diabetic nephropathy, and lupus nephritis compared with control, including clotting pathway components (fibrinogen), amyloid components, and apolipoprotein E.

To verify the laser capture microdissection/proteomic findings, kidney biopsies were stained for fibulin-1 and podocin, as representative proteins that were increased or decreased, respectively, in the fibronectin glomerulopathy kidneys. Fibulin-1 showed mild diffuse staining in the expanded mesangium of patients with fibronectin glomerulopathy (Figure 4b). No fibulin-1 staining was seen in normal control kidneys (Figure 4a), in the Kimmelstiel-Wilson nodules of diabetic nephropathy (Figure 4c), or in the wire-loop lesions of lupus nephritis (Figure 4d). It is known that fibulin-1 is present in basement membranes, ${ }^{31,32}$ consistent with the staining along the glomerular capillary walls seen in the glomeruli of diabetic nephropathy and lupus nephritis, and less intensely in normal kidneys.

Podocin is known to be expressed in the slit diaphragms of podocytes, ${ }^{33}$ and podocin staining was seen in the podocytes along the glomerular capillary loops in normal glomeruli (Figure 5a). Staining was present but less intense in the biopsies with fibronectin glomerulopathy (Figure 5b) and lupus nephritis (Figure $5 \mathrm{~d}$ ). Staining was almost negative in the glomeruli with diabetic nephropathy (Figure 5c). 


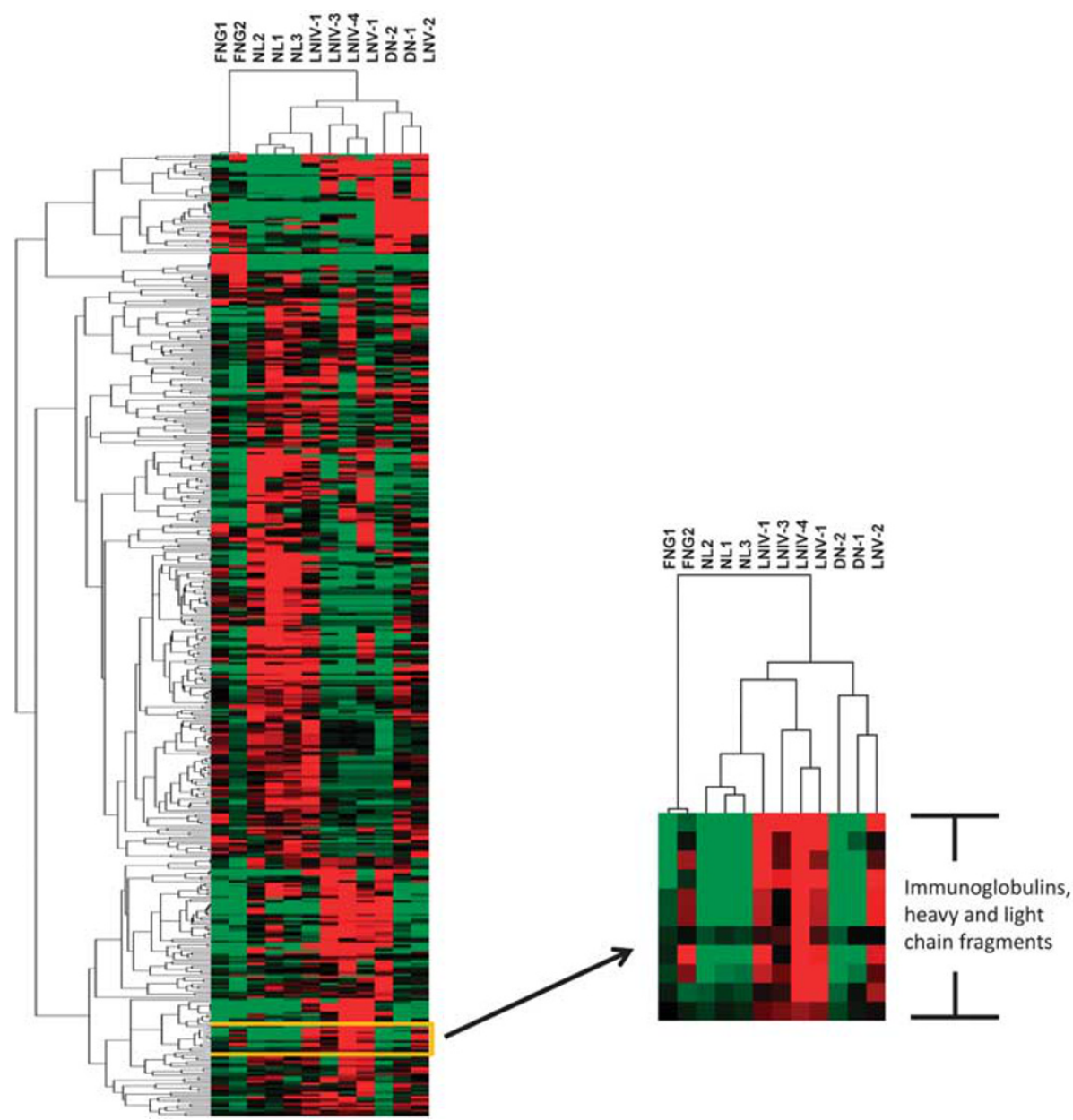

Figure 3 Heat map of glomerular proteins found in normal glomeruli, and glomeruli from patients with fibronectin glomerulopathy, diabetic nephropathy, and lupus nephritis. Each vertical bar represents a single normal individual or patient, as indicated. Each horizontal bar represents a separate protein. Red indicates relatively more abundant proteins, and green less abundant proteins. The small inset map is a section representing immunoglobulin proteins and fragments, demonstrating their presence only in the five cases of lupus nephritis.

Given the unexpected similarities in protein expression from three clinically disparate types of glomerular disease, Ingenuity Pathway Analysis was used to functionally annotate glomerular proteins from normal and diseased kidneys (Figure 6). Representative biologic functions or canonical pathways based on the differentially expressed proteins are shown. The complement system, humoral immunity, and inflammatory response pathways were increased in fibronectin glomerulopathy, diabetic nephropathy, and lupus nephritis. Metabolic pathways appeared to be significantly down-regulated in all three glomerular diseases.

The discovery that fibulin was highly represented in the lesions of fibronectin glomerulopathy prompted us to determine if there were unique single-nucleotide polymorphisms in the fibulin-1 gene that segregated with fibronectin glomerulopathy. Although a number of singlenucleotide polymorphisms were found (data not shown), none appeared to segregate with fibronectin glomerulopathy.

\section{Discussion}

The present work demonstrates the successful application of laser capture microdissection followed by mass spectrometry in the characterization of glomerular proteomes from clinical kidney biopsies. The high degree of clustering within the normal group and the fibronectin glomerulopathy group is an indication of the reproducibility of the method. There was more variability in the diabetic nephropathy and lupus nephritis patient groups; however, this is not unexpected. Kidney biopsies in diabetics are generally done in patients with atypical clinical courses, and in lupus nephritis there is a considerable spectrum of pathologic changes within individual lupus nephritis classes. Glomerular 
Table 1 Glomerular protein expression in fibronectin glomerulopathy

\begin{tabular}{|c|c|c|c|c|c|c|c|c|c|}
\hline Protein & Fibronectin glomerulopathy/ $\mathrm{N}^{\mathrm{a}}$ & $\mathrm{G}^{\mathrm{b}}$ & Diabetic nephropathy/N & $\mathrm{G}$ & Lupus nephritis IV/N & $\mathrm{G}$ & Lupus nephritis $V / \mathrm{N}$ & G & \\
\hline \multicolumn{10}{|l|}{ Matrix proteins } \\
\hline Fibronectin 1 & 14 & 665 & & & & & & & \\
\hline Fibronectin 3 & 14 & 663 & & & & & & & \\
\hline Fibronectin 8 & 14 & 668 & & & & & & & \\
\hline Fibronectin 14 & 14 & 66 & & & & & & & \\
\hline Fibulin-1 & 53 & 44 & & & & & & & \\
\hline Fibulin-1D & 76 & 67 & & & & & & & \\
\hline Fibulin-5 & 16.6 & 13.8 & & & & & & & \\
\hline Laminin $\alpha-5$ & 0.5 & 25 & & & & & & & \\
\hline Heparan sulfate proteoglycan core protein & 2 & 19 & 3 & 35 & & & 1.5 & 5 & \\
\hline \multicolumn{10}{|l|}{ Complement system } \\
\hline $\mathrm{C} 1 \mathrm{~g}$ & 13 & 7 & & & 20 & 12 & & & \\
\hline C3 & 4.4 & 33 & 37 & 39 & 10 & 109 & 13 & 120 & \\
\hline $\mathrm{C} 4 \mathrm{~B}$ & 4.2 & 15 & 7 & 19 & & & 10 & 44 & \\
\hline C5 & 18 & 12 & 30 & 13 & 22 & 14 & 67 & 41 & \\
\hline C8 & & & & & & & 13 & 5.5 & \\
\hline C9 & & & & & 2.4 & 5.7 & 5 & 23.5 & \\
\hline Factor $\mathrm{H}$ & 10 & 5 & & & & & & & \\
\hline \multicolumn{10}{|l|}{ Serum proteins } \\
\hline APOE & 12 & 7 & 30 & 13 & & & 31 & 17 & \\
\hline Amyloid P & 4.6 & 10 & 9 & 19 & & & 4.8 & 8 & \\
\hline Transthyretin & 12 & 7 & & & & & & & \\
\hline Fibrinogen $\alpha$-chain & 26 & 39 & 47 & 46 & 35 & 49 & 45 & 54 & 3 \\
\hline Fibrinogen $\beta$-chain & 40 & 32 & 62 & 30 & 56 & 41 & 67 & 41 & $\begin{array}{c}\stackrel{\sim}{*} \\
\stackrel{\tilde{O}}{*}\end{array}$ \\
\hline \multicolumn{9}{|l|}{ Podocyte proteins } & $\frac{\overline{\hat{\Theta}}}{\sqrt{0}}$ \\
\hline Podocin & 0.06 & 15 & 0.4 & 7 & 0.14 & 9.4 & 0.17 & 7 & $\frac{0}{0}$ \\
\hline Synaptopodin & 0.13 & 43 & 0.3 & 13 & 0.35 & 16.6 & & & \\
\hline A-actinin & 0.7 & 14 & 0.7 & 8 & & & & & \\
\hline Complement receptor 1 isoform $F$ precursor & 0.06 & 15 & 0.1 & 7 & 0.07 & 15 & 0.09 & 9 & \\
\hline Neprilysin & 0.16 & 41 & 0.14 & 26 & 0.21 & 30 & 0.34 & 15 & \\
\hline Tight junction protein $\mathrm{ZO}-1$ & 0.24 & 22 & 0.09 & 24 & 0.2 & 22 & 0.27 & 14 & \\
\hline MYH9 & 0.65 & 33 & & & & & & & \\
\hline \multicolumn{10}{|l|}{ Anti-oxidant proteins } \\
\hline Peroxiredoxin-5 & 0.18 & 6 & & & & & & & \\
\hline Peroxiredoxin-6 & 0.07 & 12 & 0.12 & 6 & 0.08 & 9.8 & 0.10 & 7.5 & \\
\hline Glutathione S-transferase A1 & 0.1 & 7 & & & 0.12 & 5.6 & & & \\
\hline Glutathione S-transferase A2 & 0.1 & 8 & & & 6 & 0.11 & & & \\
\hline \multicolumn{10}{|c|}{${ }^{\mathrm{a}}$ Ratio of normalized spectral counts of a protein found in a glomerular disease to the normalized spectral counts of the protein in control glomeruli. } \\
\hline
\end{tabular}


Table 2 Raw protein spectral counts for individual patients and controls

\begin{tabular}{|c|c|c|c|c|c|}
\hline \multicolumn{3}{|c|}{ Controls } & \multicolumn{2}{|c|}{ Fibronectin } & \multirow[t]{2}{*}{ Protein identification } \\
\hline 1 & 2 & 3 & 1 & 2 & \\
\hline 16 & 20 & 34 & 287 & 410 & IPI:IPI00022418.1 | FN1 isoform 1 of fibronectin \\
\hline 16 & 20 & 34 & 287 & 409 & IPI:IPI00339223.1|FN1 isoform 3 of fibronectin \\
\hline 16 & 20 & 32 & 285 & 410 & IPI:IPI00339228.1|FN1 isoform 8 of fibronectin \\
\hline 16 & 20 & 32 & 285 & 409 & IPI:IPI00855777.1 |FN1 isoform 14 of fibronectin \\
\hline 1 & 0 & 0 & 24 & 31 & IPI:IPI00296534.2 | FBLN1 isoform D of fibulin-1 \\
\hline 0 & 0 & 0 & 18 & 19 & IPI:IPI00889740.1 | FBLN1 fibulin-1 \\
\hline 0 & 0 & 0 & 7 & 5 & IPI:IPI00294615.3|FBLN5 fibulin-5 \\
\hline 48 & 51 & 61 & 33 & 19 & IPI:IPI00783665.4 | LAMA5 laminin subunit $\alpha-5$ \\
\hline 30 & 21 & 37 & 58 & 66 & IPI:IPI00024284.5 | HSPG2 basement membrane-specific heparan sulfate proteoglycan core protein \\
\hline 0 & 0 & 0 & 4 & 5 & IPI:IPI00022394.2 |C1QC complement C1q subcomponent subunit C \\
\hline 4 & 4 & 12 & 30 & 34 & IPI:IPI00783987.2 |C3 complement C3 (Fragment) \\
\hline 0 & 6 & 4 & 15 & 15 & IPI:IPI00892604.1 |C4B;C4A complement component C4B (Childo blood group) 2 \\
\hline 0 & 0 & 0 & 7 & 1 & IPI:IPI00816741.1 |C5 complement component 5 variant (Fragment) \\
\hline 0 & 0 & 0 & 4 & 2 & IPI:IPI00011261.2 | C8G complement component C8 gamma chain \\
\hline 5 & 5 & 5 & 14 & 9 & IPI:IPI00022395.1 | C9 complement component C9 \\
\hline 0 & 0 & 0 & 5 & 2 & IPI:IPI00029739.5 | CFH isoform 1 of complement factor $\mathrm{H}$ \\
\hline 0 & 0 & 0 & 7 & 2 & IPI:IPI00021842.1 | APOE apolipoprotein E \\
\hline 0 & 5 & 0 & 15 & 5 & IPI:IPI00022391.1 | APCS serum amyloid P-component \\
\hline 0 & 0 & 0 & 3 & 6 & IPI:IPI00022432.1 I TTR transthyretin \\
\hline 0 & 2 & 0 & 18 & 20 & IPI:IPI00029717.1 |FGA isoform 2 of fibrinogen $\alpha$ chain \\
\hline 0 & 0 & 0 & 10 & 19 & IPI:IPI00298497.3 | FGB fibrinogen $\beta$ chain \\
\hline 8 & 10 & 5 & 0 & 0 & IPI:IPI00014594.1 INPHS2 isoform 1 of podocin \\
\hline 34 & 20 & 28 & 7 & 0 & IPI:IPI00027258.3 | SYNPO Isoform 2 of synaptopodin \\
\hline 97 & 80 & 97 & 71 & 60 & IPI:IPI00013808. 1 | TACTN $4 \alpha$-actinin- 4 \\
\hline 7 & 0 & 2 & 0 & 0 & IPI:IPI00787286.1 | CR1 Complement component (3b/4b) receptor 1 \\
\hline 35 & 20 & 27 & 7 & 3 & IPI:IPI00247063.3 | MME neprilysin \\
\hline 24 & 20 & 18 & 9 & 2 & IPI:IPI00335824.5 | TJP1 isoform short of tight junction protein ZO-1 \\
\hline 187 & 183 & 244 & 172 & 116 & IPI:IPI00019502.3 | MYH9 isoform 1 of myosin-9 \\
\hline 6 & 3 & 5 & 2 & 0 & IPI:IPI00024915.2 | PRDX5 isoform mitochondrial of peroxiredoxin-5; mitochondrial \\
\hline 8 & 4 & 7 & 1 & 0 & IPI:IPI00220301.5 | PRDX6 peroxiredoxin-6 \\
\hline 6 & 0 & 6 & 0 & 0 & IPI:IPI00657682.2 | GSTA1 glutathione S-transferase A1 \\
\hline 7 & 0 & 6 & 0 & 0 & IPI:IPI00745233.2 | GSTA2 glutathione S-transferase A2 \\
\hline
\end{tabular}

immunohistochemistry was used to validate differential glomerular expression of selected proteins identified by the proteomic approach. Additional validation was provided by the finding that several of the differentially expressed proteins revealed by proteomic analysis are consistent with proteins known to be involved in glomerular injury, for example, complement activation products and immunoglobulins in lupus nephritis, or fibronectin in fibronectin glomerulopathy.

The most interesting findings from this study are the newly discovered changes in protein expression. This demonstrates that our approach is a robust platform that may inform disease pathogenesis. For example, despite the diversity of glomerular diseases examined, we found a number of glomerular protein changes that were common in the disease glomeruli but not the normal glomeruli, as follows:

(1) Several podocyte proteins, particularly those associated with the slit diaphragm, were significantly diminished in fibronectin glomerulopathy, diabetic nephropathy, and lupus nephritis. As all of these diseases are proteinuric, and because the attenuated podocyte proteins are important in the maintenance of the glomerular filtration barrier, decreased expression likely contributes to proteinuria. Interestingly, laser capture microdissection followed by microarray analysis of glomeruli from patients with focal segmental glomerulosclerosis showed a down-regulation of mature podocyte genes. ${ }^{5}$ It is not presently known if the loss of these podocyte proteins and genes is a nonspecific response to podocyte injury and/or proteinuria, or is a primary event in the development of proteinuria. The latter scenario would imply a common pathogenic mechanism for different diseases.

(2) Complement components were found in fibronectin glomerulopathy, diabetic nephropathy, and lupus nephritis. The presence of complement proteins in the glomeruli of lupus nephritis patients is expected, though the patterns of class IV and class $V$ are slightly different. Class IV lesions uniquely contained C1q, no C4 protein, and abundant C3 protein. The presence of C3 without C4 suggests significant alternative pathway activation, and is in agreement with our recent report of C3 consumption, but not C4 consumption, being independently associated with renal flare. ${ }^{34}$ In contrast, class $\mathrm{V}$ lesions contained no C1q but detectable C4 protein, suggesting the involvement of the lectin pathway. Other complement components were also present in class $\mathrm{V}$ lesions, including abundant C9, likely reflecting the presence of the complement membrane attack complex as previously reported. ${ }^{35}$ 

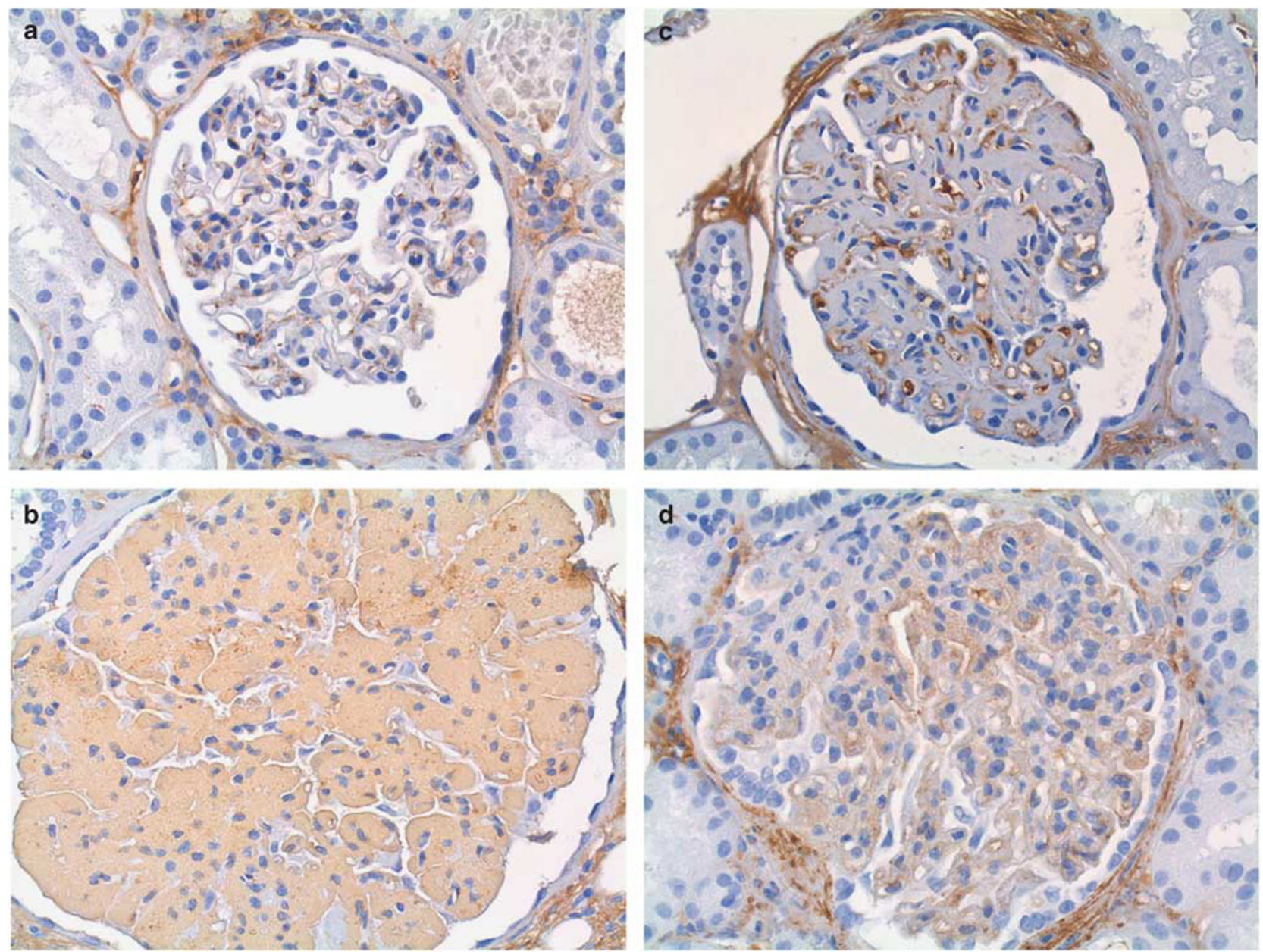

Figure 4 Immunoperoxidase staining for fibulin-1. (a) Baseline allograft biopsy with normal glomerulus shows no mesangial staining $(\times 400)$. (b) Biopsy with fibronectin glomerulopathy showing an enlarged glomerulus with mild but global mesangial staining $(\times 400)$. (c) Biopsy of patient with diabetic glomerulosclerosis. No staining in expanded mesangium. Staining along glomerular capillary wall is noted $(\times 400)$. (d) Biopsy of proliferative lupus nephritis with no staining in the mesangium.

In diabetic nephropathy, there are some data that complement may be involved in disease progression, possibly through the lectin-binding pathway, and $\mathrm{C} 3$ and the membrane attack complex (C5b-9) have been found in glomeruli (reviewed in Ostergaard et $a{ }^{36}$ ).

The presence of complement proteins in fibronectin glomerulopathy glomeruli is somewhat surprising, because immunohistochemical analysis of fibronectin glomerulopathy reveals inconsistent involvement of immunoglobulins or complement. ${ }^{13}$ Fibronectin binds $\mathrm{C} 1 \mathrm{q}$ via its collagenous tail, ${ }^{37,38}$ which could explain the presence of C1q, but this binding does not result in complement activation. ${ }^{39}$ Complement activation could occur through the alternative pathway, especially if the expression or function of complement regulators is compromised. Interestingly, a genome-wide linkage study identified a fibronectin glomerulopathy genetic susceptibility locus within the regulators of complement activation gene cluster. ${ }^{40}$ However, no studies to date have identified abnormal expression or func- tion of regulators of complement activation gene cluster proteins in patients with fibronectin glomerulopathy. ${ }^{41}$ Another possible explanation for the presence of complement proteins in fibronectin glomerulopathy is through glomerular complement expression. This has been demonstrated for C3 by in situ hybridization in glomeruli from lupus nephritis patients, but not from healthy controls. ${ }^{42}$ Finally, this could simply represent nonspecific trapping of complement proteins, but if so this is significantly more than in normal glomeruli (see raw spectral counts, Table 2). The presence of complement proteins in the glomeruli of fibronectin glomerulopathy raises the possibility that complement-mediated damage may be involved in this nonimmune glomerular lesion. A recent proteomic evaluation of chronic allograft nephropathy also unexpectedly found complement pathway activation and reached a similar conclusion. ${ }^{43}$ Limiting complement activation may therefore improve clinical outcomes in diseases not previously thought to involve clinically relevant complement activation. 

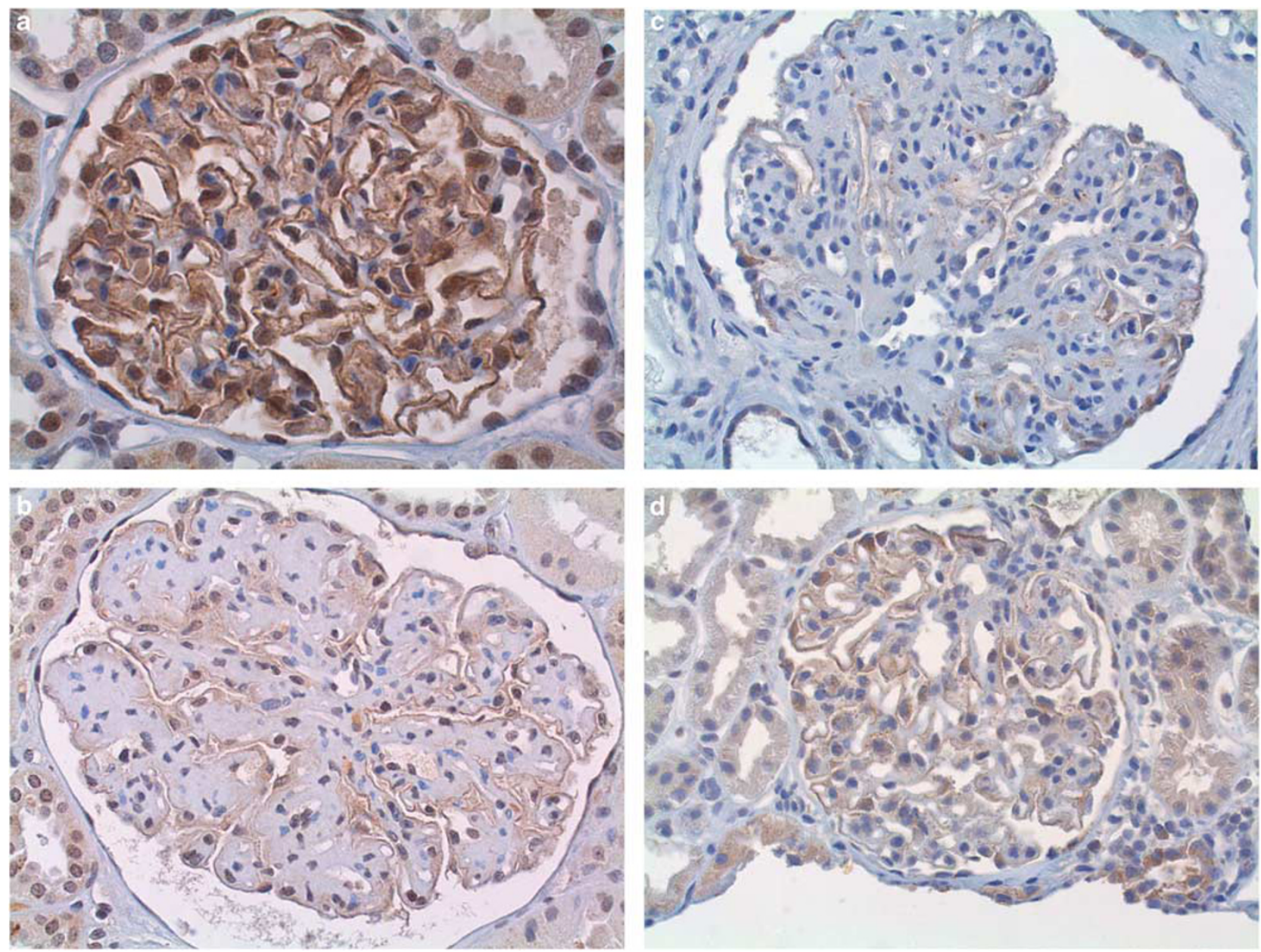

Figure 5 Immunoperoxidase staining for podocin. (a) Baseline allograft biopsy with normal glomerulus shows staining of podocytes along capillary wall $(\times 400)$. (b) Biopsy with fibronectin glomerulopathy showing an enlarged glomerulus with weak staining in the capillary wall $(\times 400)$. (c) Biopsy of patient with diabetic glomerulosclerosis. No staining in podocytes $(\times 400)$. (d) Biopsy of proliferative lupus nephritis, with weak staining in podocytes $(\times 400)$.

(3) Anti-oxidant proteins were conspicuously under-represented in fibronectin glomerulopathy, diabetic nephropathy, and lupus nephritis glomeruli, but the proteomic approach cannot distinguish between a primary defect in anti-oxidant production, or a secondary loss of these proteins. Nonetheless, a reduction in glomerular anti-oxidants leaves the tissue open for oxidant injury.

(4) Pathway analysis based on the differentially expressed proteins suggested that compared with normal, metabolic pathways were suppressed in all three types of glomerular disease. This suggests that any type of glomerular injury may attenuate metabolism of glomerular cells, but more importantly, indicates that metabolomic evaluation of glomerular disease may uncover novel mechanisms of injury and repair.

To further demonstrate the utility of laser capture microdissection followed by glomerular proteomics, we studied fibronectin glomerulopathy in more detail. This uncommon glomerular disease results from the deposition of excessive matrix material in glomeruli. The hypothesis that fibronectin glomerulopathy is mechanistically related to abnormal glomerular accumulation of fibronectin is based mainly on evidence from immunohistochemical staining, which has limitations. Immunohistochemical staining can only test constituents for which specific antibodies are available, and can be falsely positive due to nonspecific trapping of antibodies, or falsely negative if antigens are blocked. By isolating glomeruli from kidney biopsies of affected patients, and doing unbiased proteomic analysis of the recovered glomerular proteins, we confirmed that fibronectin is the predominant protein in the lesions of fibronectin glomerulopathy glomeruli. In addition to several common pathways of glomerular injury activated in fibronectin glomerulopathy described above, we found considerable deposition of fibulin in these glomeruli. Among the diseases examined, fibulin accumulation appears to be unique to fibronectin glomerulopathy because it is not present in normal glomeruli, diabetic nephropathy, or lupus nephritis. Interestingly, fibronectin 

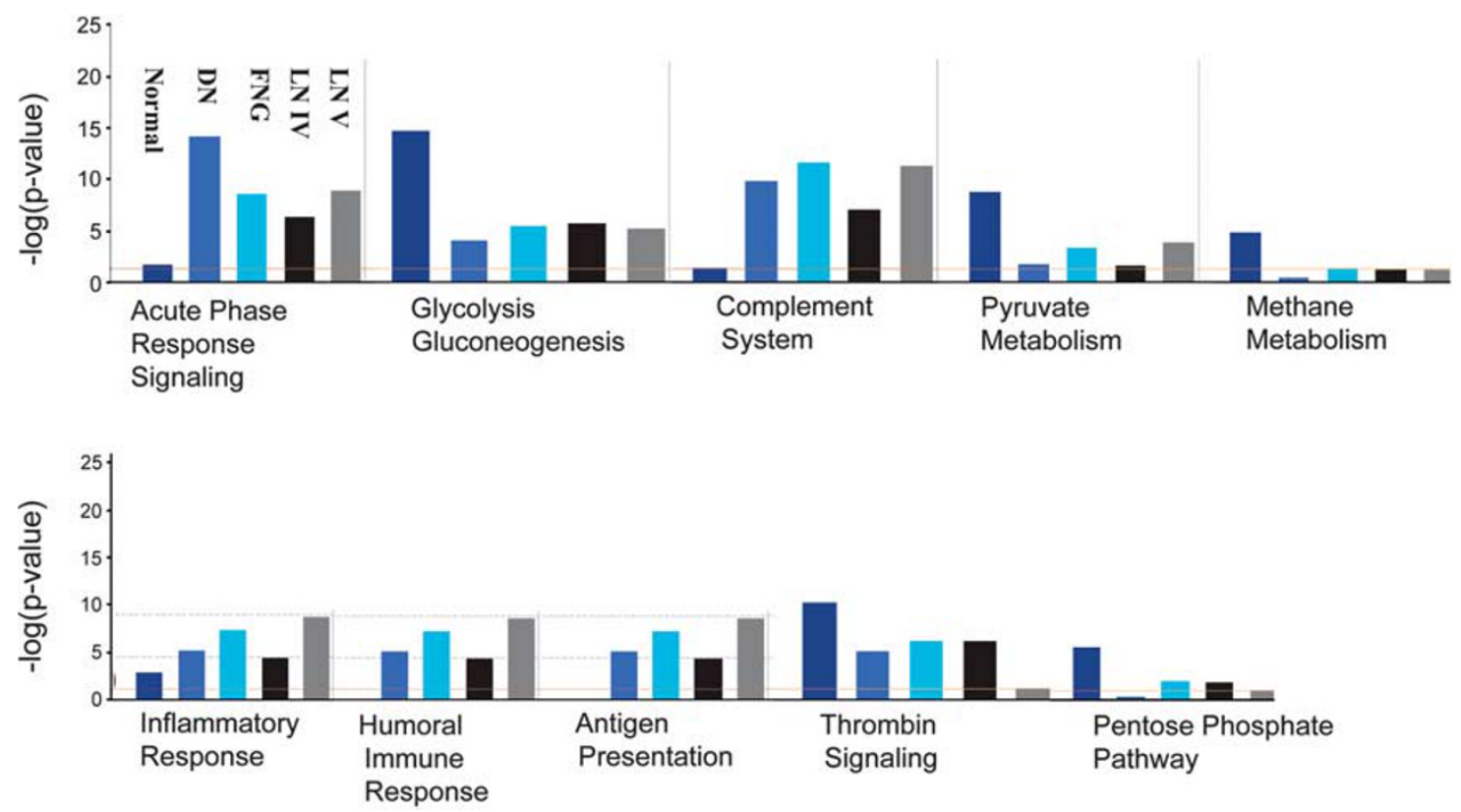

Figure 6 Representative biologic functions and pathways affected by fibronectin glomerulopathy, diabetic nephropathy, and lupus nephritis. Ingenuity Pathway Analysis was done based on differential protein expression in normal glomeruli, or glomeruli with fibronectin glomerulopathy, diabetic nephropathy, or lupus nephritis. The bars representing controls and the different disease states, as indicated, were assigned a probabilistic significance score shown as a $P$-value (y axis) for each pathway or function. The orange line represents the threshold for detection.

binds to fibulin-1 but not fibulin-5. ${ }^{31,41,44,45}$ The fibulins appear to regulate several of the molecular activities of fibronectin. For example, fibronectin is important in cell-matrix interactions, and facilitates cell attachment, cell spreading, and cell migration. ${ }^{46}$ Fibronectin also regulates the cytoskeleton, cellular morphology, and differentiation. ${ }^{30}$ Fibulin-1 downregulates fibronectin-facilitated cell spreading, adhesion, and motility. ${ }^{47}$ These effects may occur because fibulin-1 inhibits fibronectin-mediated extracellular signal-regulated kinase activation. ${ }^{47}$ Although fibulin-5 does not directly bind to fibronectin, it binds to the same $\beta 1$ integrins as fibronectin $(\alpha 5 \beta 1, \alpha 4 \beta 1)$, and like fibulin-1, blocks fibronectinmediated cell spreading, motility, and proliferation. ${ }^{48}$

The role of fibulins plus fibronectin in the pathogenesis of glomerular injury in fibronectin glomerulopathy is not yet known; however, fibulins have been associated with other types of kidney disease. Specifically, fibulin-1 was identified as a candidate permeability factor in focal segmental glomerulosclerosis, ${ }^{49}$ perhaps because it interferes with cell adhesion, and could therefore disrupt the podocyte-basement membrane relationship. Gene profiling of autosomal dominant polycystic kidney disease tissue showed markedly increased fibulin mRNA expression, and the protein was demonstrated in epithelial cells lining cysts. ${ }^{50}$ Fibulin-1 has been shown to co-localize with ADAMTS-1 and increase its catalytic activity ${ }^{51}$ It is conceivable that in fibronectin glomerulopathy, the accumulated fibronectin helps localize fibulins to glomeruli, and the fibulins inhibit cell spreading, adhesion, and motility, disrupting mesangial and/or podocyte function and facilitating the development of proteinuria.

In summary, the present study shows that the application of laser capture microdissection followed by liquid chromatography-tandem mass spectrometry analysis of kidney biopsies can provide large-scale quantitative proteomic profiles that may be important for characterizing glomerular diseases as well as determining mechanisms of disease pathogenesis. The ability to more clearly understand underlying molecular events critical to disease expression may open avenues for novel therapeutic interventions.

\section{Acknowledgement}

This work was supported in part by DK077331 (BHR) and RR025755 (MF).

\section{Disclosure/conflict of interest}

The authors declare no conflict of interest.

\section{References}

1 Edgley AJ, Gow RM, Kelly DJ. Laser-capture microdissection and pressure catapulting for the analysis of gene expression in the renal glomerulus. Meth Mol Biol 2010;611:29-40. 
2 Kohda Y, Murakami H, Moe OW, et al. Analysis of segmental renal gene expression by laser capture microdissection. Kidney Int 2000;57:321-331.

3 Schmid H, Henger A, Cohen CD, et al. Gene expression profiles of podocyte-associated molecules as diagnostic markers in acquired proteinuric diseases. J Am Soc Nephrol 2003;14:2958-2966.

4 Rudnicki M, Eder S, Perco P, et al. Gene expression profiles of human proximal tubular epithelial cells in proteinuric nephropathies. Kidney Int 2007;71: 325-335.

5 Bennett MR, Czech KA, Arend LJ, et al. Laser capture microdissection-microarray analysis of focal segmental glomerulosclerosis glomeruli. Nephron Exp Nephrol 2007;107:e30-e40.

6 Peterson KS, Huang JF, Zhu J, et al. Characterization of heterogeneity in the molecular pathogenesis of lupus nephritis from transcriptional profiles of laser-captured glomeruli. J Clin Invest 2004;113:1722-1733.

7 Potthoff SA, Sitek B, Stegbauer J, et al. The glomerular proteome in a model of chronic kidney disease. Proteomics Clin Appl 2008;2:1127-1139.

$8 \mathrm{Xu}$ BJ, Shyr Y, Liang X, et al. Proteomic patterns and prediction of glomerulosclerosis and its mechanisms. J Am Soc Nephrol 2005;16:2967-2975.

9 Miyamoto M, Yoshida Y, Taguchi I, et al. In-depth proteomic profiling of the normal human kidney glomerulus using two-dimensional protein prefractionation in combination with liquid chromatographytandem mass spectrometry. J Proteome Res 2007;6:3680-3690.

10 Sitek B, Potthoff S, Schulenborg T, et al. Novel approaches to analyse glomerular proteins from smallest scale murine and human samples using DIGE saturation labelling. Proteomics 2006;6:4337-4345.

11 Yoshida Y, Miyamoto M, Taguchi I, et al. Human kidney glomerulus proteome and biomarker discovery of kidney diseases. Proteomics Clin Appl 2008;2:420-427.

12 Gemperle O, Neuweiler J, Reutter FW, et al. Familial glomerulopathy with giant fibrillar (fibronectin-positive) deposits: 15-year follow-up in a larger kindred. Am J Kidney Dis 1996;28:668-675.

13 Strom EH, Banfi G, Krapf R, et al. Glomerulopathy associated with predominant fibronectin deposits: a newly recognized hereditary disease. Kidney Int 1995;48:163-170.

14 Hildebrandt F, Strahm B, Prochoroff A, et al. Glomerulopathy associated with predominant fibronectin deposits: exclusion of the genes for fibronectin, villin and desmin as causative genes. Am J Med Gen 1996;63:323-327.

15 Mazzucco G, Maran E, Rollino C, et al. Glomerulonephritis with organized deposits: a mesangiopathic, not immune complex-mediated disease? A pathologic study of two cases in the same family. Hum Path 1992;23:63-68.

16 Sekiguchi K, Siri A, Zardi L, et al. Differences in domain structure between human fibronectins isolated from plasma and from culture supernatants of normal and transformed fibroblasts. Studies with domainspecific antibodies. J Biol Chem 1985;260:5105-5114.

17 Zhao HL, Lai FM, Tong PC, et al. Clinicopathologic characteristics of nodular glomerulosclerosis in Chinese patients with type 2 diabetes. Am J Kidney Dis 2004;44:1039-1049.

18 Ding L, Zhao M, Zou W, et al. Mycophenolate mofetil combined with prednisone for diffuse proliferative lupus nephritis: a histopathological study. Lupus 2004;13:113-118.

19 Oomura A, Nakamura T, Arakawa M, et al. Alterations in the extracellular matrix components in human glomerular diseases. Virchows Arch A Pathol Anat Histopathol 1989;415:151-159.

20 Tuttle SE, Sharma HM, Bay WH, et al. A unique familial lobular glomerulopathy. Arch Pathol Lab Med 1987;111:726-731.

$21 \mathrm{Xu} \mathrm{H}$, Freitas MA. MassMatrix: a database search program for rapid characterization of proteins and peptides from tandem mass spectrometry data. Proteomics 2009;9:1548-1555.

22 Tucci M, Lombardi L, Richards HB, et al. Overexpression of interleukin-12 and T helper 1 predominance in lupus nephritis. Clin Exp Immunol 2008;154:247-254.

$23 \mathrm{Xu} \mathrm{H}$, Freitas MA. Monte Carlo simulation-based algorithms for analysis of shotgun proteomic data. J Proteome Res 2008;7:2605-2615.

$24 \mathrm{Xu} \mathrm{H}$, Freitas MA. A mass accuracy sensitive probability based scoring algorithm for database searching of tandem mass spectrometry data. BMC Bioinformatics 2007;8:133.

25 Lundgren DH, Hwang SI, Wu L, et al. Role of spectral counting in quantitative proteomics. Exp Rev Proteomics 2010;7:39-53.

26 Carvalho PC, Hewel J, Barbosa VC, et al. Identifying differences in protein expression levels by spectral counting and feature selection. Genet Mol Res 2008;7:342-356.

27 Liu H, Sadygov RG, Yates III JR. A model for random sampling and estimation of relative protein abundance in shotgun proteomics. Anal Chem 2004;76: 4193-4201.

28 Zhang B, Chambers MC, Tabb DL. Proteomic parsimony through bipartite graph analysis improves accuracy and transparency. J Proteome Res 2007;6:3549-3557.

29 Zhang B, VerBerkmoes NC, Langston MA, et al. Detecting differential and correlated protein expression in label-free shotgun proteomics. J Proteome Res 2006;5:2909-2918.

30 Castelletti F, Donadelli R, Banterla F, et al. Mutations in FN1 cause glomerulopathy with fibronectin deposits. Proc Natl Acad Sci USA 2008;105:2538-2543.

31 de Vega S, Iwamoto T, Yamada Y. Fibulins: multiple roles in matrix structures and tissue functions. Cell Mol Life Sci 2009;66:1890-1902.

32 Singh U, Sun T, Larsson T, et al. Expression and functional analysis of fibulin-1 (Fbln1) during normal and abnormal placental development of the mouse. Placenta 2006;27:1014-1021.

33 Roselli S, Gribouval O, Boute N, et al. Podocin localizes in the kidney to the slit diaphragm area. Am J Pathol 2002;160:131-139.

34 Birmingham DJ, Irshaid F, Nagaraja $\mathrm{HN}$, et al. The complex nature of serum C3 and C4 as biomarkers of lupus renal flare. Lupus 2010;19:1272-1280.

35 Lai KN, Lo ST, Lai FM. Immunohistochemical study of the membrane attack complex of complement and S-protein in idiopathic and secondary membranous nephropathy. Am J Pathol 1989;135:469-476.

36 Ostergaard J, Hansen TK, Thiel S, et al. Complement activation and diabetic vascular complications. Clin Chim Acta 2005;361:10-19.

37 Rovin BH, McKinley AM, Birmingham DJ. Can we personalize treatment for kidney diseases? Clin J Am Soc Nephrol 2009;4:1670-1676. 
38 Bing DH, Almeda S, Isliker $\mathrm{H}$, et al. Fibronectin binds to the C1q component of complement. Proc Natl Acad Sci USA 1982;79:4198-4201.

39 Pearlstein E, Sorvillo J, Gigli I. The interaction of human plasma fibronectin with a subunit of the first component of complement, C1q. J Immunol 1982; 128:2036-2039.

40 Vollmer M, Jung M, Ruschendorf F, et al. The gene for human fibronectin glomerulopathy maps to 1q32, in the region of the regulation of complement activation gene cluster. Am J Hum Genet 1998;63:1724-1731.

41 Vollmer M, Kremer M, Ruf R, et al. Molecular cloning of the critical region for glomerulopathy with fibronectin deposits (GFND) and evaluation of candidate genes. Genomics 2000;68:127-135.

42 Miyazaki M, Abe K, Koji T, et al. Intraglomerular C3 synthesis in human kidney detected by in situ hybridization. J Am Soc Nephrol 1996;7:2428-2433.

43 Nakorchevsky A, Hewel JA, Kurian SM, et al. Molecular mechanisms of chronic kidney transplant rejection via large-scale proteogenomic analysis of tissue biopsies. J Am Soc Nephrol 2010;21:362-373.

44 Godyna S, Mann DM, Argraves WS. A quantitative analysis of the incorporation of fibulin-1 into extra- cellular matrix indicates that fibronectin assembly is required. Matrix Biol 1995;14:467-477.

45 Balbona K, Tran H, Godyna S, et al. Fibulin binds to itself and to the carboxyl-terminal heparin-binding region of fibronectin. J Biol Chem 1992;267:20120-20125.

46 Miyamoto S, Katz BZ, Lafrenie RM, et al. Fibronectin and integrins in cell adhesion, signaling, and morphogenesis. Ann NY Acad Sci 1998;857:119-129.

47 Twal WO, Czirok A, Hegedus B, et al. Fibulin-1 suppression of fibronectin-regulated cell adhesion and motility. J Cell Sci 2001;114:4587-4598.

48 Lomas AC, Mellody KT, Freeman LJ, et al. Fibulin-5 binds human smooth-muscle cells through alpha5beta1 and alpha4beta1 integrins, but does not support receptor activation. Biochem J 2007;405:417-428.

49 Musante L, Candiano G, Bruschi M, et al. Characterization of plasma factors that alter permeability to albumin within isolated glomeruli. Proteomics 2002;2:197-205.

50 Schieren G, Rumberger B, Klein M, et al. Gene profiling of polycystic kidneys. Nephrol Dial Transplant 2006;21:1816-1824.

51 Lee NV, Rodriquez-Manzaneque JC, Thai SN, et al. Fibulin-1 acts as a cofactor for the matrix metalloprotease ADAMTS-1. J Biol Chem 2005;280:34796-34804. 